\title{
Aggregation Effects on Pigment Coatings: Pigment Red 179 as a Case Study
}

\author{
Francesco Muniz-Miranda, ${ }^{\dagger}$ Pierpaolo Minei, ${ }^{\ddagger}$ Luca Contiero, ${ }^{\S}$ Frédéric Labat, $^{\dagger}$ (i) Ilaria Ciofini, ${ }^{\dagger}$
}

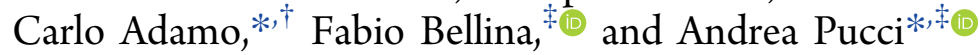

†École Nationale Supérieure de Chimie de Paris and PSL Research University, CNRS, Institute of Chemistry for Life and Health Sciences (i-CLeHS), FRE 2027, 11, rue Pierre et Marie Curie, F-75005 Paris, France

${ }^{\ddagger}$ Deparment of Chemistry and Industrial Chemistry, University of Pisa, Via Giuseppe Moruzzi 13, 56124 Pisa, Italy

${ }^{\S}$ Cromology Italia S.P.A., Via 4 Novembre 4, 55016 Porcari, Lucca, Italy

\section{Supporting Information}

ABSTRACT: Here, we have studied, with a combined experimental and computational approach, the effect of the crystal environment and aggregation on the electronic properties of Pigment Red 179, which affect both its color and optical energy gap. Spectra acquired in the near-infrared and visible range of energies suggest that this molecule is indeed a "cool" dye, which can be employed as a red pigment that provides effective color coverage to different substrates without contributing to their heating during light irradiation. Spectra acquired on different polymer mixtures at different pigment concentrations (i.e., 2.5-10 wt \%) suggest that absorption features depend on chromophoric arrangements promoted by the strong intermolecular $\pi-\pi$ interactions. Calculations,
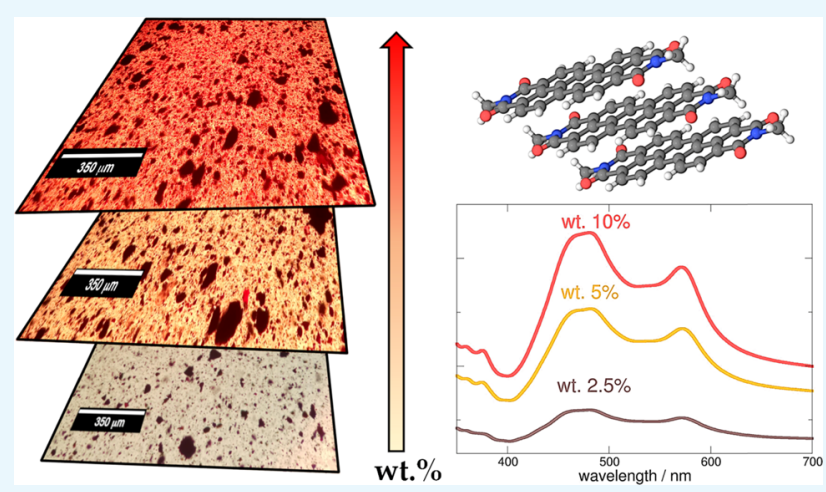
performed at the time-dependent density functional theory level, allowed to both attribute the nature of the electronic transitions causing the observed spectra involved and understand the effect of the environment. Indeed, the visible spectra of the pigment is dominated by two localized transitions, with negligible charge transfer for both a dye monomer and dimer either in vacuum or acetonitrile solution. Instead, models including the crystal environment of the pigment show the presence of a highwavelength $S_{1} \leftarrow S_{0}$ charge transfer transition between two adjacent molecules, in quantitative agreement with the experimental absorption energy of the crystal pigment.

\section{INTRODUCTION}

Nowadays, it is widely accepted that human activities strongly contribute to the urban heat island (UHI) effect, with metropolitan areas significantly warmer than surrounding country areas. ${ }^{1-3}$ Urban areas are densely populated, thus contributing to average and peak energy demands especially during summer and straining energy resources mainly based on fossil fuels still today. In EU countries, buildings account for $40 \%$ of final energy demand and $32 \%$ of $\mathrm{CO}_{2}$ emission, including residential, industrial, and service sectors. ${ }^{4}$ With the stringent need for increasing energy efficiency and reducing greenhouse gas emission, improving the energy performance of Europe's building stock is mandatory. Nevertheless, the EU renovation rate $(1.2 \%$ in EU) is slower than expected and Kyoto and Paris agreement targets reasonably appear out of reach as also mentioned in the 2010/31/EU Directive, which states that each new building should be made "nearly zero energy" from 2020 onward. An accessible solution to comply with the EU regulation and to mitigate the UHI effect is mimicking the NIR transparent and reflective features of plants and leaves by coating the building surfaces with cool pigments. $^{5,6}$

When the sunlight shines onto a material, part of the radiation is absorbed, thus heating its surface. This is caused by $52 \%$ of the electromagnetic spectrum of light composed by the near-infrared (NIR) window (i.e., from 700 to $2500 \mathrm{~nm}$ ) that promotes chemical bond vibration, thus provoking temperature increase. ${ }^{7}$ Heat is then transported by conduction into the material, elevating the temperature of the internal side by convection. It is already reported that cool pigments and coatings are able to reduce the heating effect of sunlight with corresponding temperature differences $>10{ }^{\circ} \mathrm{C}$, therefore reducing the electricity demand and saving energy required to downgrade the UHI phenomenon. 8 " Cool surfaces are measured considering the amount of NIR radiation they reflect (solar reflectance) and how rapidly and efficiently they dissipate heat (thermal emittance). ${ }^{10}$ NIR reflective pigments

Received: August 31, 2019

Accepted: November 4, 2019

Published: November 18, 2019 
are categorized as cool pigments, which are nowadays dominated by inorganic compounds such as chromium and rare-earth oxides, thanks to their higher refractive indexes, but affected by evident toxicity issues. ${ }^{8}$ Titanium dioxide is also reported as an effective reflector of vis-NIR light but it strongly absorbs UV light, thus also promoting potential degradation of the organic coating. Therefore, organic cool pigments are an object of growing interest owing to their safe properties, low cost, and very good processability and dispersibility in most of the polymeric formulations.

Among all available organic pigments, perylene bis-imide derivatives represent the most investigated cool components of organic coatings thanks to their light and thermal stability, dispersion properties in polymers, and color modulation in the visible region thanks to the different functionalizations at the peripheral positions of the perylene nucleus. ${ }^{1-16}$

The NIR transparent and potential reflective properties of perylenes have been investigated in the literature and attributed to different parameters, such as pigment particle size, crystallinity, and dipole moment of the chromophoric supramolecular structure. ${ }^{17,18}$

Nevertheless, certain cool perylene bisimide pigments still lack full investigation aimed to determine the influence of their aggregation extent on the vis-NIR optical features of the derived polymer coatings. These properties are thus of paramount relevance for current and prospective applications and are deeply rooted into the molecular geometry, environment, and electronic structure of ground and excited states. Color, in particular, may depend in general on the molecular environments around the chromophores and, as it has been shown, on their aggregation state. ${ }^{18}$ Therefore, a deep understanding of the effects induced by the aggregation on the optoelectronic properties of dyes is needed to fine-tune them for application where light-matter interactions are relevant. In this regard, computational modeling at the quantum level (QM) can give unique insights on the mechanism behind aggregation-induced optical processes, both qualitative and quantitative. ${ }^{19,20}$ However, most investigations of periodic molecular crystals performed at the QM level are limited to the simulation of the electronic ground state, while excited states are often simulated using a userdefined cluster of molecules extracted from the solid-state structure and treated often at the time-dependent (TD) density functional theory level (DFT). This approach, while at times successful, does not consider the effect of an extended periodic environment properly, thus resulting in limited predictive and explanatory power. To properly include shortand long-range electrostatic effects, recently some of us developed a charge embedding technique enabling to take into account the effect of the exact Madelung crystal field potential on a small region of interest treated at the quantum mechanical (QM) level, by a finite array of fitted point charges. This computationally affordable model has been proven to correctly simulate the environmental effects in absorption and emission spectra of molecular crystals.

In this work, we have combined these theoretical tools with experimental investigations to understand the optical features upon aggregation of the $N, N^{\prime}$-bis-(methyl)perylene-3,4,9,10tetracarboxyldiimide dye (hereafter called Pigment Red 179 or PR179, see Figure 1), a perylene-derivative dye of significant current and prospective relevance. Different coatings were prepared as a function of the pigment content and their vis-

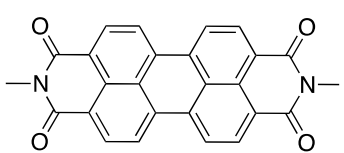

Figure 1. Chemical structure of $N, N^{\prime}$-bis-(methyl)perylene-3,4,9,10tetracarboxyldiimide (Pigment Red 179).

NIR optical features studied in terms of their supramolecular structure.

We have organized the paper as follows. In the Experimental Details section, we report the experimental optical setup used to probe of PR179 in both solution and solid states. In the Computational Details section, we describe the adopted DFT and TD-DFT procedure. In the Results and Discussion section, we present the collected chemical and physical data analyzing and commenting them. Finally, in the Concluding Remarks section, we discuss the main findings of this paper and how they can be relevant for the field of light-matter interaction.

\section{EXPERIMENTAL DETAILS}

Materials. Pigment Red 179 was obtained from BASF and utilized as received. Disperbyk 2015 (solution of a structured acrylate copolymer with pigment-affinic groups) was obtained from BYK USA Inc. Polidisp (acrylic acid esters and styrene copolymer dispersion, $50 \%$ solid content, glass transition temperature of $18{ }^{\circ} \mathrm{C}$ ) was received from Resiquimica. Linear low-density polyethylene (LLDPE, Dowlex SC 2107, Melt flow index, $190{ }^{\circ} \mathrm{C} / 2.16 \mathrm{~kg}, 2.3 \mathrm{~g} / 10 \mathrm{~min}, d=0.917 \mathrm{~g} / \mathrm{cm}^{3}$ ) was supplied by Dow Plastics. The remaining chemicals were obtained from Sigma-Aldrich.

Coating Preparation. To a desired amount of Pigment Red 179, Disperbyk 2015 (1:1 by weight with respect to the pigment) and $2 \mathrm{~g}$ of the Polidisp acrylic dispersion polymer were added and mechanically mixed at room temperature until a homogeneous mixture was obtained. After the addition with $500 \mu \mathrm{L}$ of deionized water, the resultant paint was applied to Leneta checkerboard charts or to cellulose acetate films (3 M AF4300, Write-on Transparency Film) using a ZUA 2000 Universal Applicator (Zehntner Testing Instruments) to obtain 60-70 $\mu \mathrm{m}$ thick films after complete water evaporation. In the case of coatings based on linear low-density polyethylene (LLDPE), a desired amount of Pigment Red 179 was dispersed under vigorous stirring in a 5 wt \% LLDPE solution in toluene. After evaporation of the solvent, the blend was compressionmolded at $180^{\circ} \mathrm{C}$ for $5 \mathrm{~min}$ into a thin film of about $200 \mu \mathrm{m}$ thickness.

Methods. UV-vis-NIR reflectance measurements of black- and white-coated surfaces were recorded over the wavelength range of $300-2500 \mathrm{~nm}$ by using an Agilent Cary 5000 spectrophotometer equipped with a $150 \mathrm{~mm}$ integration sphere and according to ASTM E903-12 (the standard test method for solar absorptance, reflectance, and transmittance of materials using integrating spheres). NIST standards were utilized as primary standards for the calibration of instrument.

$\mathrm{X}$-ray powder diffraction (XRD) was performed using a Bruker D2 Phaser diffractometer $(30 \mathrm{kV}, 10 \mathrm{~mA})$ operating in Bragg-Brentano geometry $(\theta-\theta$ scan mode) and equipped with a one-dimensional Lynxeye detector. Ni-filtered $\mathrm{Cu} \mathrm{K} \alpha$ radiation was used. Data were collected in the scan range of 4$70^{\circ}$ in $2 \theta$, with a scan step of $0.018^{\circ}$ and count time of $0.5 \mathrm{~s} /$ 
step. Data were processed through the software Diffrac.Eva (Bruker AXS).

The thickness of the coating layer was measured with a CM1S dial indicator (Borletti, Milan, Italy) with ruby movement bearing.

A commercially available $100 \mathrm{~W}$ IR lamp (Kerbl, Buchbach, Germany) was used for the heating tests. The specimens were irradiated at a distance of $20 \mathrm{~cm}$ for $15 \mathrm{~min}$, and then the temperature measured by means of an FLIR E6 infrared thermocamera (FLIR, Wilsonville, OR).

The optical microscope analysis was accomplished on a Reichert Polyvar optical microscope with crossed polarizers. Particle analysis was performed using the public domain ImageJ $1.52 \mathrm{k}$ software version image analyzer program developed by the National Institutes of Health and available on the internet at https://imagej.nih.gov/ij/index.html.

\section{COMPUTATIONAL DETAILS}

Nonembedded Calculations. PR179 has been studied here by means of DFT structural ground-state optimization in vacuo with the $\mathrm{B} 3 \mathrm{LYP}^{21,22}$ functional using the Gaussian software (development version: ES64L-GDVRevI.12+ ${ }^{23}$ ). To model dispersion forces, we adopted Grimme's D3-BJ correction $^{24}$ (keyword GD3BJ in the Gaussian software). All relaxed structures are actual potential energy minima, as checked by harmonic frequency calculations (i.e., all normal modes have a positive frequency). Using the relaxed groundstate structure, vertical excitations have been computed at the TD-DFT level, with the PBE0, ${ }^{25,26} \mathrm{LC}-\mathrm{PBE},{ }^{27}$ and CAM$\mathrm{B} \mathrm{LYP}^{28}$ functionals: these exchange-correlation functionals have been chosen to span both global hybrid (PBE0) and range-separated hybrid ones (LC-PBE, CAM-B3LYP), which often provide a more reliable description of through space charge-transfer excitations. Calculations at the PBE0 and LCPBE level are reported in Figures S2 and S3 of the Supporting Information. These calculations were performed both in vacuum and simulating interactions with the nonprotic polar solvent (acetonitrile, ACN), using an implicit solvent model (polarizable continuum model, the Gaussian default IEFPCM). ${ }^{29}$ The results of solvent calculations are reported in Figure S2 of the Supporting Information.

Periodic and Embedded TDDFT Calculations. All periodic calculations have been carried out with the Crystal $17^{30}$ code at the B3LYP-D3 level, as for the isolated species, along with a modified all-electron $6-31+\mathrm{G}(\mathrm{d}, \mathrm{p})$ basis set omitting the most diffuse functions. Numerical DFT integration has been performed considering 75 radial points and 974 angular points, ensuring an error on the integrated electron density to an accuracy of $10^{-5}$ e per unit cell. The Coulomb and exchange series were truncated with threshold values of $10^{-7}, 10^{-7}, 10^{-7}, 10^{-7}$, and $10^{-21}$. A tolerance of $10^{-7}$ $\mathrm{au}$ has been used for the convergence of the self-consistent field procedure. Cell parameters and atomic positions have been relaxed during geometry optimization. Convergence was determined from the root mean square and absolute value of the largest component of the forces and displacements, considering default values $(0.00045$ and 0.00067 au for the forces, and 0.0018 and 0.0027 au for the displacements, respectively). The crystalline structure of PR179 belongs to the monoclinic $P 21 / c$ space group, with flat perylenetetracarboxylicdiimide portions packed in stacks through $\pi-\pi$ interactions, with experimental lattice parameters of $a=3.874(1) \AA$, $b=15.580(2) \AA$, and $c=14.597(2) \AA, \beta=97.65(2)^{\circ}$. The optimized lattice parameters obtained are $a=3.715 \AA$, $b=$ $15.535 \AA, c=14.193 \AA$, and $\beta=100.50^{\circ}$. In addition, the flat portions of neighboring molecules are found $3.75 \AA$ apart, with respect to an experimental value of $3.87 \AA$. To create a more cubiclike box, we adopted a $4 \times 1 \times 1$ monoclinic supercell, resulting in a simulation box of volume $\sim 14.9 \times 15.5 \times 14.2$ $\AA^{3}$, shown in Figure S1 of the Supporting Information.

Starting from the optimized crystal structure obtained, a large supercell was built and partitioned into three zones, according to the SC-Ewald approach. ${ }^{31}$ At the inner first zone treated at the QM level, four different clusters with either one, two, three or four PR179 molecules have been considered. The intermediate second zone consisted of point charges fixed at the value of the Mulliken atomic charges of the periodic calculation, while the point charge values of the outer third zone have been adjusted to impose the exact Madelung potential of the infinite periodic system on the first two zone sites. Further details are available in ref 20 as well as in some of our previous works. ${ }^{32-36}$ Vertical TD-DFT calculations with the aforementioned models (embedded monomer, dimer, trimer, and tetramer of PR179) have been carried out, thus including the effects of the crystal environment. The embedded dimer model was also used to test the dependence of computed spectra on the intermolecular distance. All calculations (both embedded and nonembedded) have been carried out using the Pople's $6-31+G(d, p)$ basis set, and dispersion forces were modeled employing Grimme's D3-BJ correction. $^{24}$

\section{RESULTS AND DISCUSSION}

Experimental Investigations of Pigment Red 179 (PR179) Dispersions. Properties of PR179 Dispersions on Opaque Substrates. Vis-NIR reflectance spectra recorded on PR179 dispersion over the white portion of Leneta (L) checkerboard chart (Figure 2a) display a reflectance pattern affected by pigment content, i.e., particularly pronounced up to $1200 \mathrm{~nm}$, and in agreement with the absorption characteristics of the chromophoric unit. ${ }^{12}$ Notably, the $10 \%$ of reflectance reduction due to the pigment coating was more pronounced by increasing the pigment content from 2.5 to $5 \mathrm{wt} \%$. Conversely, no evident variations actually occurred for the coating containing the highest PR179 content (10 wt \%), thus suggesting that a sufficient coverage of the white checkboard was reached with the intermediate $5 \mathrm{wt} \%$ concentration.

Moreover, reflectance values as high as $80 \%$ recorded from white substrates suggested that Pigment Red 179 is relatively NIR transparent. ${ }^{37-39}$ This characteristic was confirmed by inspecting the reflectance behavior of pigment coatings over black substrates (Figure 2b). Relatively low reflectances were recorded in agreement with the NIR transparent features of Pigment Red 179. Nevertheless, a worthwhile increase of the overall reflectance in the 700-1600 nm wavelength window occurred with pigment concentration. Notably, on passing from 2.5 to $10 \mathrm{wt} \%$ of the pigment concentration, $75 \%$ of light reflection was recovered. This behavior suggests that Pigment Red 179 could also behave as an NIR reflective molecule, in agreement with certain symmetrically substituted perylene derivatives. ${ }^{11}$

NIR reflectivity was also associated to a cooling effect of the surface by monitoring the temperature enhancement of the black coated and uncoated substrates after irradiation with an IR lamp for $15 \mathrm{~min}$ (Figure 3). 

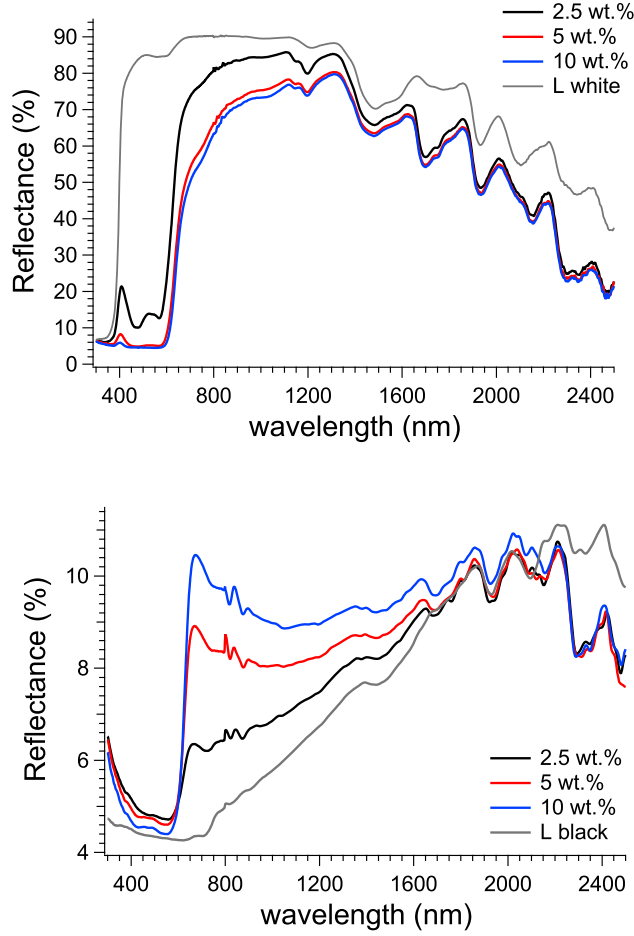

Figure 2. Vis-NIR reflectance spectra of Pigment Red 179 dispersions over white (a) and black (b) Leneta (L) checkerboard charts. (a)

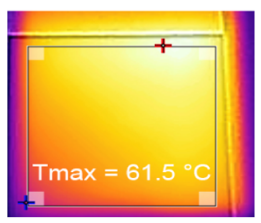

(b)

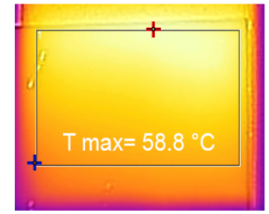

(c)

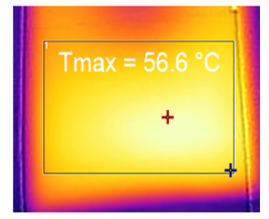

Figure 3. Thermographs of the (a) uncoated, (b) $5 \mathrm{wt} \%$, and (c) 10 wt \% Pigment Red 179 coated black checkboards with the maximum temperature reached after irradiation with a $100 \mathrm{~W}$ IR lamp for 15 $\min$.

It was worth noting that the maximum temperature reached by the uncoated black surface (Figure 3a) was recorded as the highest at $61.5^{\circ} \mathrm{C}$, i.e., 2.7 and $4.9^{\circ} \mathrm{C}$ higher than those coated by the 5 wt \% (Figure 3b) and the 10 wt \% (Figure 3c) Pigment Red 179 dispersions, respectively. This performance, while on one hand appears less pronounced than that of ultimate paints with chilling effects higher than $10-15{ }^{\circ} \mathrm{C}$, on the other hand confirms the cooling characteristics of the investigated symmetrically substituted perylene bisimide derivative.

Aimed at determining the origin of the cool features of Pigment Red 179, the dispersions were analyzed by XRD investigations at room temperature (Figure 4).

The XRD pattern of the pigment dispersions displayed sharp reflections attributed to the crystalline content that is dominated by an evident elevation caused by the amorphous phase contribution, which appears predominant at the lower $\theta$ region and mostly attributed to the acrylic amorphous polymer coating. Notably, the diffraction profiles in the higher $\theta$ regions $\left(\theta>25^{\circ}\right)$ showed crystalline contributions that are attributed to self-assembled and well-ordered microstructures promoted

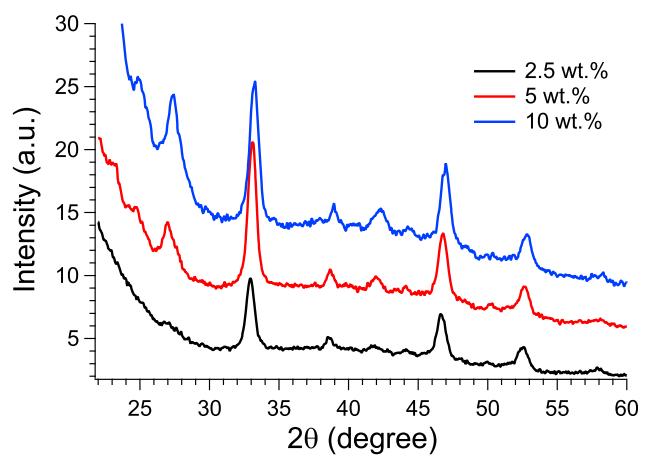

Figure 4. XRD spectra of the Pigment Red 179 dispersions at different dye concentrations.

by the formations of intermolecular $\pi-\pi$ interactions. ${ }^{16,19}$ It is worth noting that a considerable fraction of crystalline Pigment Red 179 is present at all investigated concentrations with an evident and progressive increase of the reflection intensities particularly evidenced on passing from the 2.5 to the $5 \mathrm{wt} \%$ pigment concentration. Unfortunately, the presence of the strong amorphous contribution provided by the polymer covered most of the crystalline peaks of the pigment at the $\theta$ region lower than $22^{\circ}$. This issue flanked by experimental systematic errors made a quantitative assessment of the crystalline degree inaccurate. Nevertheless, the favorable morphology and crystallinity of $\pi-\pi$ pigment stacked structures possibly explain enhancement in the NIR reflectivity.

Properties of PR179 Dispersions on Transparent Substrates. Pigment Red 179 dispersion was also deposited on transparent cellulose acetate polymer films aimed at determining the morphology of the pigment aggregates as well as the spectroscopic features in the visible region. Optical micrographs reported in Figure 5 show the presence of a broad distribution of microsized pigment particles and large aggregates.

The average diameter calculated on passing from 2.5 to 10 wt $\%$ solid dispersions remained almost unchanged (about 3-5 $\mu \mathrm{m}$ ), whereas the variation of light intensity and sample color appeared evident for pigment concentrations higher than $5 \mathrm{wt}$ $\%$. This behavior agreed with the results gathered from reflectance experiments of pigment dispersions over white substrates (Figure 2a), whose coverage resulted mostly complete by using the dispersion with 5-10 wt \% Pigment Red 179 concentration. It is also well reported that diffuse reflection intensity depends on the number of particle boundaries. ${ }^{40}$ By increasing pigment concentration, the coating layer contains a large number of small micrometer-sized particles with high surface area that possibly contribute to the higher NIR reflectance due to the enhanced number of boundaries.

The color of the Pigment Red 179 is mostly addressed to its planar conjugated $\pi$-electron system, which causes strong absorption in the visible region. The methyl substituent at the periphery of the molecule is reported to be responsible for the energetic position of the absorption maximum. ${ }^{41}$

Perylene bisimides exhibit the electronic $0-0,0-1$ vibrational peaks and high energy $0-2$ featureless shoulder peaks in the region of 410-450 nm, which are usually fused together in a broadened absorption band in chromophoric aggregates in solution or in the solid state. In the case of Pigment Red 179 dispersions over cellulose acetate films, this broad absorption 


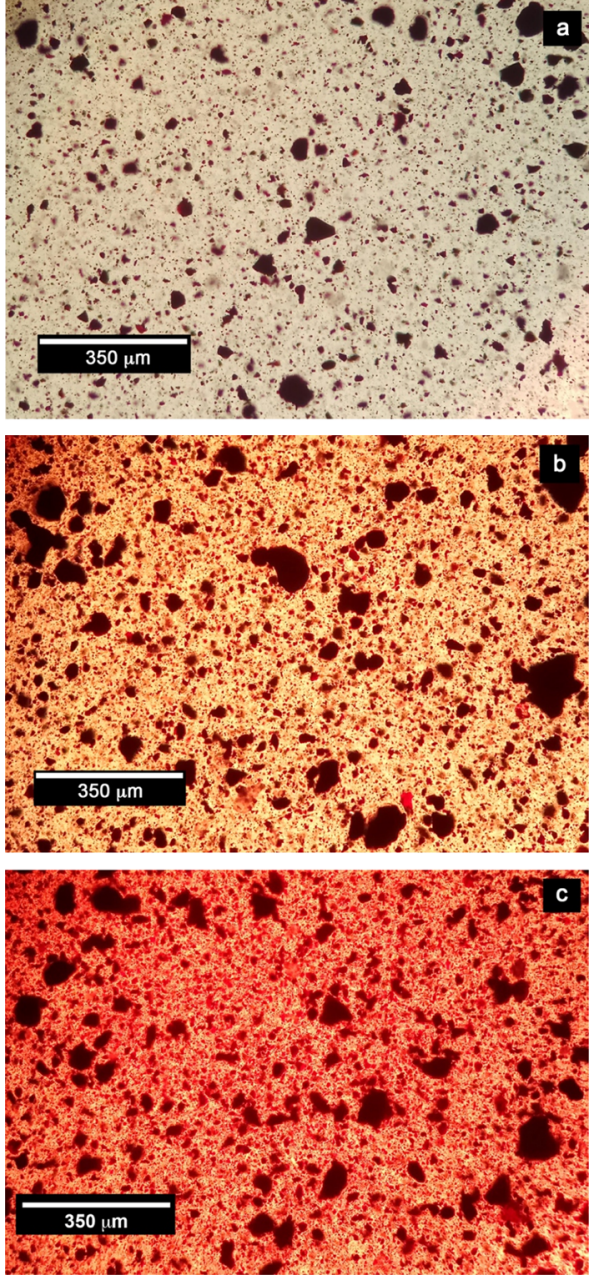

Figure 5. Optical micrographs of Pigment Red 179 dispersions over cellulose acetate films at different pigment contents. Scale bar $=350$ $\mu \mathrm{m}$. (a) $2.5 \mathrm{wt} \%$; (b) $5 \mathrm{wt} \%$; (c) $10 \mathrm{wt} \%$ pigment.

band is located at about $480-490 \mathrm{~nm}$ and flanked by more growth of shoulder peaks at the longer wavelengths of 570$580 \mathrm{~nm}$. This feature is in agreement with the formation of the typical face-to-face chromophoric arrangements promoted by the strong intermolecular $\pi-\pi$ interactions, as analogously reported in the main literature. ${ }^{42-45}$ Notably, the ratio between the absorbances of these two contributions did not seem affected by pigment concentration, thus suggesting that the formation of large aggregates already occurred at the lowest 2.5 wt \% content, as also evidenced by optical microscopy in Figure 5a. The band at lower energies did not prevail over the molecular one even in a polyethylene matrix (Figure 6, yellow curve), which is considered the less compatible one with organic pigments and its apolar and aliphatic nature is well known to strongly promote the formation of large-scale chromophoric aggregates. ${ }^{46-50}$

Computational Analysis of Pigment Red 179 (PR179) Assemblies. Aimed at understanding the effect of the aggregation extent of Pigment Red 179 molecules on their optical characteristics, a computational analysis was carried out. We report in Figure 7 (left) the computed absorption spectrum for an isolated PR179 molecule. The effect of solvent on the band shape profile and transition character is negligible, as clearly shown by comparison with calculations in ACN reported in Figure S2 of the Supporting Information.

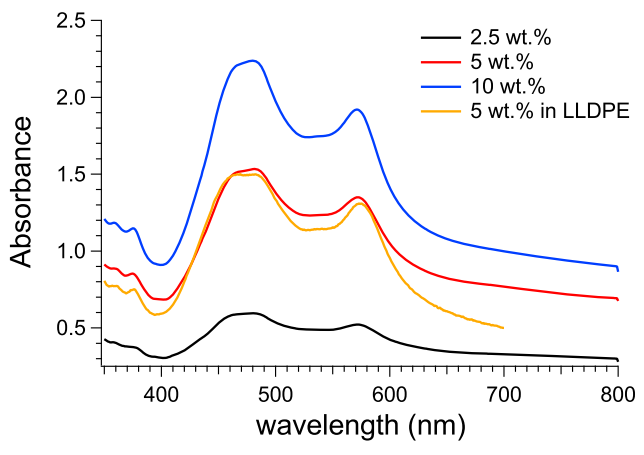

Figure 6. Absorption spectra of Pigment Red 179 dispersions over cellulose acetate transparent films as a function of pigment content and 5 wt \% Pigment Red 179/LLDPE film (yellow line).

The spectrum is dominated by two main transitions, all of a localized character with a negligible intramolecular chargetransfer character. Both transitions are basically $\pi-\pi^{*}$ excitations corresponding to a HOMO $\rightarrow$ LUMO excitation for transition I and to a HOMO - $1 \rightarrow$ LUMO for II (see bottom left side of Figure 7). This assignment is consistent among the three levels of theory tested here, with only a shift in energy in the computed spectra due to their different Hartree-Fock exchange contents (up to 100 and $\sim 63 \%$ for long interelectronic distances in LC-PBE and CAM-B3LYP, respectively, and $25 \%$ for the global hybrid PBE0). To model the effect of $\pi-\pi$ intermolecular interactions, a minimal model represented by a dimer of PR179 molecules (refer to Computational Methods section for details) was first considered and the results are reported in Figure 7 (right side). As can be seen in Figure 7, bottom right panel, the nature of the main transitions changes (I of the HOMO $\rightarrow$ LUMO type, and II of the HOMO $-1 \rightarrow$ LUMO + 1 type) but retains their localized character, without any significant charge transfer, neither intra nor intermolecular. To have an estimate of the charge-transfer distance associated to these transitions, we employed the $D_{\text {ct }}$ index, ${ }^{51}$ which is basically a measure of the hole-electron distance due to the photoexcitation. We found $D_{\text {ct }}$ values of 0.06 and $0.09 \AA$ for excitations I and II, respectively: these small values, much smaller than an average covalent bond distance, are fully consistent with the notion of a localized transition (i.e., a transition with no charge transfer involved).

CAM-B3LYP vertical excitations show absorption in the blue-cyan region of the visible spectrum (for the isolated pigment and dimer model), while the absorption energies computed at the PBE0 level of theory are significantly redshifted with optical gaps in the orange region of the visible spectrum, as expected due to the reduced amount of HartreeFock exchange. ${ }^{52-56}$

The spectrum of the isolated dimer computed at CAMB3LYP and LC-PBE levels shows no qualitative difference with respect to that of the monomer but a slight red-shift of around $50 \mathrm{~nm}$. The PBE0 spectra show larger differences.

Nonetheless, to correctly simulate the crystalline environment effect on the optical spectra, as mentioned in the introduction, long-range electrostatic effects have to be included. To this end, embedded TD-DFT calculations were performed and the corresponding simulated spectrum at the CAM-B3LYP level is reported in Figure 8 in the case of the dimer model, the minimal one to check the existence of intermolecular CT transitions. The orbital component of the 

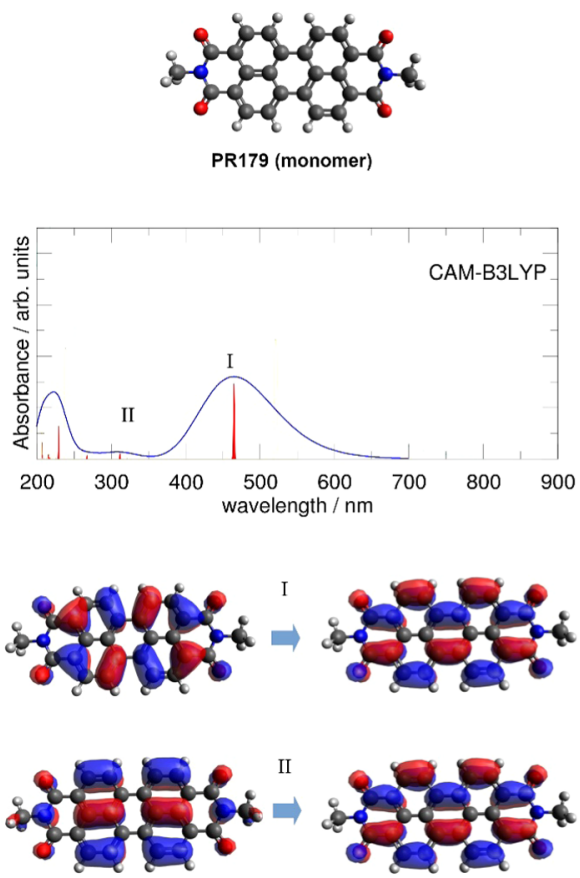
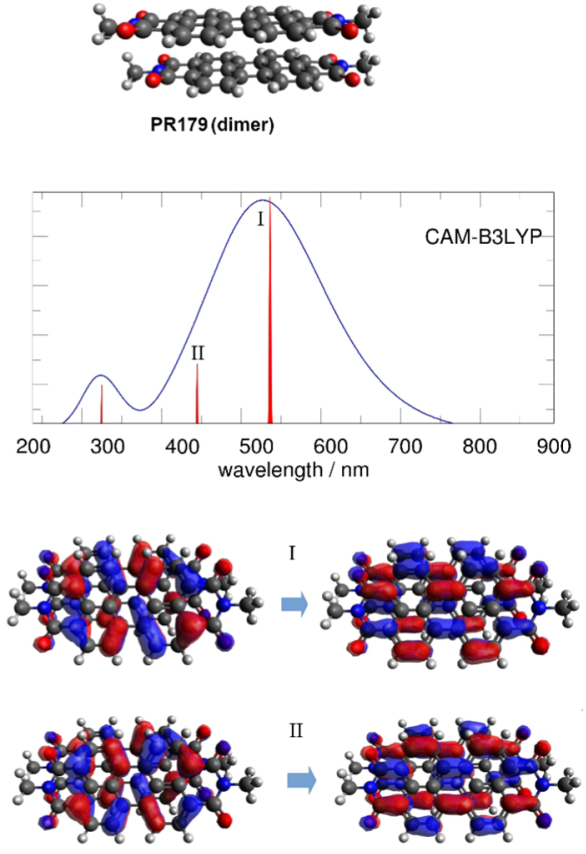

Figure 7. Computed UV-vis spectra of PR179 monomer (left) and isolated dimer (right). Vertical red spikes represent computed vertical excitation transitions, while blue lines are spectra obtained by Gaussian convolution using a full width half-height of $0.33 \mathrm{eV}$. The orbitals involved in transitions I and II are reported for CAM-B3LYP. No energy/wavelength scaling has been employed.

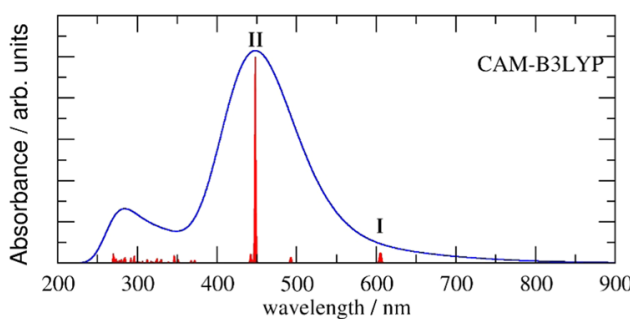

I
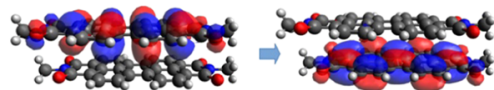

II

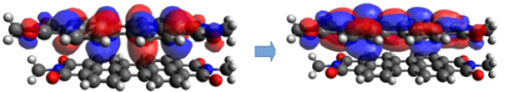

Figure 8. Computed UV-vis spectrum of PR179 dimer embedded in the electrostatic field of the crystal. Red spikes represent actual transitions, while the blue line is the spectrum obtained by Gaussian convolution using a full width half-height of $0.33 \mathrm{eV}$. The orbitals involved in transitions I and II are reported for CAM-B3LYP. No energy/wavelength scaling has been employed.

first (I) and the second (II) transitions are reported in Figure 8. Contrary to the calculations performed in the absence of the crystalline potential, here we can clearly see the presence of a transition ( $\mathrm{I}$ at low energies in red region when using the CAM-B3LYP functional), which shows a clear intermolecular charge-transfer character (the $D_{c t}$ index ${ }^{51}$ estimates a chargetransfer distance of $\sim 3.91 \AA$, consistent with the intermolecular distance between PR179 molecules). This transition involved the HOMO and LUMO orbitals localized on different molecules, whereas the second one (II) is of the HOMO $\rightarrow$ LUMO + 1 type and does not show any charge-transfer character. The predicted transition energies (at TD-CAM-
B3LYP level of theory) are $\lambda_{\mathrm{I}}=605 \mathrm{~nm}$ and $\lambda_{\mathrm{II}}=448 \mathrm{~nm}$, corresponding to energies of 2.04 and $2.77 \mathrm{eV}$. These absorption energies are in good agreement with the experimental ones (2.13 and $2.69 \mathrm{eV}$, respectively, see Figure 6).

Calculations performed on embedded trimer and tetramer clusters confirm the intermolecular charge transfer nature of the 1st transition: the orbital involved in it belongs to two adjacent molecules, without the creation of an extended exciton: these are reported in the Supporting Information (Figures S4 and S5).

To investigate how both energies and intensity of the two bands are affected by packing, we decided to monitor the variation in oscillator strength and transition energies of the two bands as a function of the intermolecular distance in the presence of the embedding potential. Changing the intermolecular distance significantly affects the oscillator strength of transition I, as reported in Figure 9. Here, it has be pinpointed that distances much smaller than the experimental equilibrium value (of about $\sim 3.86 \AA$ ) might have little physical meaning, for at least two reasons: (1) it is experimentally unfeasible to compress the crystal system so far as to reach such short intermolecular contacts; (2) for very short intermolecular distances, the orbital character of the $S_{0} \rightarrow S_{1}$ and $S_{0} \rightarrow S_{2}$ transitions may change drastically, giving rise to a discontinuous behavior of both energy and oscillator strength; considering this caveat, we report them anyway in Figure 9 (from 2.5 to $4 \AA$ ) for the sake of completeness. When the distance decreases to about $15 \%$ with respect to the equilibrium one, the UV-vis peak due to transition I matches the absorption of transition II. To match the relative height of the two peaks, as observed experimentally in Figure 6, a shrinkage of the intermolecular distance of about $6 \%$ is needed. The oscillator strength of a transition is in fact proportional to 
Transition I
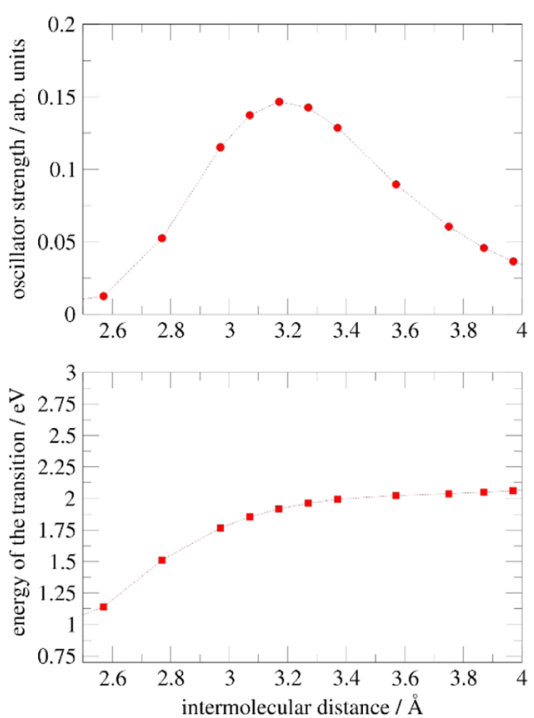

Transition II
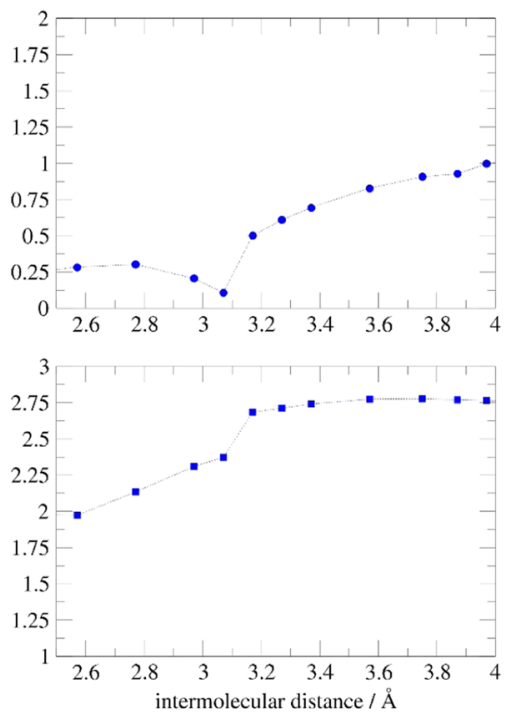

Figure 9. Oscillator strength (CAM-B3LYP level of theory) and energies of transitions I and II, as a function of the intermolecular distance.

its probability of occurrence, and, consequently, the calculations show that the intensity of the intermolecular charge transfer increases when the intermolecular distance decreases.

While the energy of transition I remains remarkably stable when passing from intermolecular distances between $\sim 3.1$ and $\sim 4.0 \AA$ (corresponding to energy values of 1.85 and $2.06 \mathrm{eV}$, respectively), the energy of transition II is instead more affected by the change in this parameter, and, in particular, has an abrupt change between 3.2 and $3.1 \AA$. In fact, the energy of transition II decreases significantly with compression of the embedded dimer model, with values spanning the interval between $2.37 \mathrm{eV}(d=3.1 \AA)$ and $2.76 \mathrm{eV}(d=4.0 \AA)$. This can be qualitatively related to the experimentally observed red-shift of the band ascribed to transition II from $\sim 460 \mathrm{~nm}(2.69 \mathrm{eV})$ to $\sim 490 \mathrm{~nm}(2.53 \mathrm{eV})$ when increasing the pigment concentration, as shown in Figure 6. The combined rise of transition I and red-shift of transition II make the pigment darker when the concentration is increased corresponding to a locally more dense (closer) packing of the dimers. The results gathered from the computational investigations strictly evidence how the optical features of Pigment Red 179 are strongly affected by its aggregation extent. As far as concentration increases in the supporting polymer matrix, a compact and crystalline-like structure governed by $\pi-\pi$ stacking interactions grows, thus inducing the progressive emersion of the low-energy absorption CT band that, in turn, contributes to the overall color of the pigment.

\section{CONCLUDING REMARKS}

In this work, we investigate, by means of a combined approach of density functional theory calculations and vis-NIR experiments, the effects due to aggregation on the optical properties of Pigment Red 179, particularly on its color and NIR reflective features. Experimental spectra show that the pigment coverage and NIR reflective features are strongly dependent on the chromophore content. Notably, on passing from 2.5 to $10 \mathrm{wt} \%$ pigment concentration, $75 \%$ of light reflection was recovered in the 700-1600 nm wavelength window, which, in turn, produces a cooling effect of about $5{ }^{\circ} \mathrm{C}$ of the black coated surface. Calculations addressed this evidence to be due to an intermolecular charge-transfer excitation, which becomes more probable as the molecules get closer in agreement with their X-ray diffraction spectra. This work provides new insights on the aggregation-induced absorption and on the development of modern paints that offer a colored coverage to the different substrates without contributing to their heating during light exposure.

\section{ASSOCIATED CONTENT}

\section{Supporting Information}

The Supporting Information is available free of charge on the ACS Publications website at DOI: 10.1021/acsomega.9b02819.

Optimized crystal structure of the perylene crystal (S1), computed UV-vis spectra of PR179 assemblies (S2) and computed UV-vis spectra of PR179 dimer at three levels of theory embedded in the electrostatic field of the crystal (S3), energy, intensity (PDF)

\section{AUTHOR INFORMATION}

\section{Corresponding Authors}

*E-mail: carlo.adamo@chimie-paristech.fr (C.A.).

*E-mail: andrea.pucci@unipi.it (A.P.).

ORCID

Frédéric Labat: 0000-0002-1967-2769

Ilaria Ciofini: 0000-0002-5391-4522

Fabio Bellina: 0000-0002-4939-7008

Andrea Pucci: 0000-0003-1278-5004

\section{Notes}

The authors declare no competing financial interest.

\section{ACKNOWLEDGMENTS}

This work was supported by the Tuscany region POR FESR 2014-2020 COOLSUN - New NIR-reflective "cool" organic pigments. F.M.-M. gratefully acknowledge Federica Maschietto's preliminary calculations on the perylene systems. This project has received funding from the European Research Council (ERC) under the European Union's Horizon 2020 
Research and Innovation Program (grant agreement no. 648558 STRIGES CoG).

\section{REFERENCES}

(1) Muscio, A. The solar reflectance index as a tool to forecast the heat released to the urban environment: Potentiality and assessment issues. Climate 2018, 6, 12.

(2) Sarrat, C.; Lemonsu, A.; Masson, V.; Guedalia, D. Impact of urban heat island on regional atmospheric pollution. Atmos. Environ. 2006, 40, 1743-1758.

(3) Chakraborty, T.; Lee, X. A simplified urban-extent algorithm to characterize surface urban heat islands on a global scale and examine vegetation control on their spatiotemporal variability. Int. J. Appl. Earth Obs. Geoinf. 2019, 74, 269-280.

(4) Energy efficiency in Europe - Overview of Policies and Good Practices; European Energy Network, 2014.

(5) Llado, D.; Perez, P.; Urban, J. F. Cool solution for the coatings industry: improving performance by evaluating reflectance and pigment chemistry. Eur. Coat. J. 2017, 3, 74-79.

(6) Akbari, H.; Pomerantz, M.; Taha, H. Cool surfaces and shade trees to reduce energy use and improve air quality in urban areas. Sol. Energy 2001, 70, 295-310.

(7) Jose, S.; Joshy, D.; Narendranath, S. B.; Periyat, P. Recent advances in infrared reflective inorganic pigments. Sol. Energy Mater. Sol. Cells 2019, 194, 7-27.

(8) Synnefa, A.; Santamouris, M.; Apostolakis, K. On the development, optical properties and thermal performance of cool colored coatings for the urban environment. Sol. Energy 2007, 81, 488-497.

(9) Yaghoobian, N.; Kleissl, J. Effect of reflective pavements on building energy use. Urban Climate 2012, 2, 25-42.

(10) Kaur, B.; Quazi, N.; Ivanov, I.; Bhattacharya, S. N. Nearinfrared reflective properties of perylene derivatives. Dyes Pigm. 2012, 92, 1108-1113.

(11) Mazhar, M.; Abdouss, M.; Gharanjig, K.; Teimuri-Mofrad, R. Synthesis, characterization and near infra-red properties of perylenebisimide derivatives. Prog. Org. Coat. 2016, 101, 297-304.

(12) Würthner, F. Perylene bisimide dyes as versatile building blocks for functional supramolecular architectures. Chem. Commun. 2004, $1564-1579$

(13) Carlotti, M.; Gullo, G.; Battisti, A.; Martini, F.; Borsacchi, S.; Geppi, M.; Ruggeri, G.; Pucci, A. Thermochromic polyethylene films doped with perylene chromophores: Experimental evidence and methods for characterization of their phase behaviour. Polym. Chem. 2015, 6, 4003-4012.

(14) Donati, F.; Pucci, A.; Cappelli, C.; Mennucci, B.; Ruggeri, G. Modulation of the optical response of polyethylene films containing luminescent perylene chromophores. J. Phys. Chem. B 2008, 112, $3668-3679$.

(15) Raj, M. R.; Margabandu, R.; Mangalaraja, R. V.; Anandan, S. Influence of imide-substituents on the $\mathrm{H}$-type aggregates of perylene diimides bearing cetyloxy side-chains at bay positions. Soft Matter 2017, 13, 9179-9191.

(16) Kaur, B.; Bhattacharya, S. N.; Henry, D. J. Interpreting the near-infrared reflectance of a series of perylene pigments. Dyes Pigm. 2013, 99, 502-511.

(17) Meymand, F. M.; Mazhar, M.; Abdouss, M. Investigation of substituent effect on cool activity of perylene bisimide pigments. $J$. Coat. Technol. Res. 2019, 16, 439-447.

(18) Wang, W.; Sun, X.; Qu, J.; Xie, X.; Qi, Z.; Hong, D.; Jing, S.; Zheng, D.; Tian, Y.; Ma, H.; Yu, S.; Ma, J. Aggregation-induced visible light absorption makes reactant 1,2-diisocyanoarenes act as photosensitizers in double radical isocyanide insertions. Phys. Chem. Chem. Phys. 2017, 19, 31443-31451.

(19) Presti, D.; Wilbraham, L.; Targa, C.; Labat, F.; Pedone, A.; Menziani, M. C.; Ciofini, I.; Adamo, C. Understanding AggregationInduced Emission in Molecular Crystals: Insights from Theory. J. Phys. Chem. C 2017, 121, 5747-5752.
(20) Wilbraham, L.; Adamo, C.; Labat, F.; Ciofini, I. Electrostatic Embedding To Model the Impact of Environment on Photophysical Properties of Molecular Crystals: A Self-Consistent Charge Adjustment Procedure. J. Chem. Theory Comput. 2016, 12, 3316-3324.

(21) Lee, C.; Yang, W.; Parr, R. G. Development of the ColleSalvetti Correlation-energy Formula into a Functional of the Electron Density. Phys. Rev. B 1988, 37, 785-789.

(22) Becke, A. D. Density-functional Thermochemistry. III. The Role of Exact Exchange. J. Chem. Phys. 1993, 98, 5648-5652.

(23) Frisch, M. J.; Trucks, G. W.; Schlegel, H. B.; Scuseria, G. E.; Robb, M. A.; Cheeseman, J. R.; Scalmani, G.; Barone, V.; Petersson, G. A.; Nakatsuji, H.; Li, X.; Caricato, M.; Marenich, A. V.; Bloino, J.; Janesko, B. G.; Gomperts, R.; Mennucci, B.; Hratchian, H. P.; Ortiz, J. V.; Izmaylov, A. F.; Sonnenberg, J. L.; Williams; Ding, F.; Lipparini, F.; Egidi, F.; Goings, J.; Peng, B.; Petrone, A.; Henderson, T.; Ranasinghe, D.; Zakrzewski, V. G.; Gao, J.; Rega, N.; Zheng, G.; Liang, W.; Hada, M.; Ehara, M.; Toyota, K.; Fukuda, R.; Hasegawa, J.; Ishida, M.; Nakajima, T.; Honda, Y.; Kitao, O.; Nakai, H.; Vreven, T.; Throssell, K.; Montgomery, J. A., Jr.; Peralta, J. E.; Ogliaro, F.; Bearpark, M. J.; Heyd, J. J.; Brothers, E. N.; Kudin, K. N.; Staroverov, V. N.; Keith, T. A.; Kobayashi, R.; Normand, J.; Raghavachari, K.; Rendell, A. P.; Burant, J. C.; Iyengar, S. S.; Tomasi, J.; Cossi, M.; Millam, J. M.; Klene, M.; Adamo, C.; Cammi, R.; Ochterski, J. W.; Martin, R. L.; Morokuma, K.; Farkas, O.; Foresman, J. B.; Fox, D. J. Gaussian 16, Wallingford, CT, 2016.

(24) Grimme, S.; Ehrlich, S.; Goerigk, L. Effect of the damping function in dispersion corrected density functional theory. J. Comput. Chem. 2011, 32, 1456-65.

(25) Adamo, C.; Barone, V. Toward reliable density functional methods without adjustable parameters: The PBE0 model. J. Chem. Phys. 1999, 110, 6158-6169.

(26) Ernzerhof, M.; Scuseria, G. E. Assessment of the Perdew-BurkeErnzerhof exchange-correlation functional. J. Chem. Phys. 1999, 110, 5029-5036.

(27) Henderson, T. M.; Izmaylov, A. F.; Scalmani, G.; Scuseria, G. E. Can short-range hybrids describe long-range-dependent properties? J. Chem. Phys. 2009, 131, No. 044108.

(28) Yanai, T.; Tew, D.; Handy, N. A new hybrid exchangecorrelation functional using the Coulomb-attenuating method (CAMB3LYP). Chem. Phys. Lett. 2004, 393, 51-57.

(29) Tomasi, J.; Mennucci, B.; Cammi, R. Quantum mechanical continuum solvation models. Chem. Rev. 2005, 105, 2999-3093.

(30) Dovesi, R.; Erba, A.; Orlando, R.; Zicovich-Wilson, C. M.; Civalleri, B.; Maschio, L.; Rerat, M.; Casassa, S.; Baima, J.; Salustro, S.; Kirtman, B. Wiley Interdiscip. Rev.: Comput. Mol. Sci. 2018, 8, No. e1360.

(31) Klintenberg, M.; et al. Accurate Crystal Fields for Embedded Cluster Calculations. Comput. Phys. Commun. 2000, 131, 120-128.

(32) Wilbraham, L.; Louis, M.; Alberga, D.; Brosseau, A.; Guillot, R.; Ito, F.; Labat, F.; Métivier, R.; Allain, C.; Ciofini, I. Revealing the origins of mechanically-induced fluorescence changes in organic molecular crystals. Adv. Mater. 2018, 30, No. 1800817.

(33) Presti, D.; Labat, F.; Pedone, A.; Frisch, M. J.; Hratchian, H. P.; Ciofini, I.; Menziani, M. C.; Adamo, C. Computational protocol for modeling thermochromic molecular crystals: salicylidene aniline as a case study. J. Chem. Theory Comput. 2014, 10, 5577-5585.

(34) Presti, D.; Labat, F.; Pedone, A.; Frisch, M. J.; Hratchian, H. P.; Ciofini, I.; Menziani, M. C.; Adamo, C. Modeling Emission Features of Salicylidene Aniline Molecular Crystals: a QM/QM' Approach. J. Comput. Chem. 2016, 37, 861-870.

(35) Presti, D.; Pedone, A.; Ciofini, I.; Labat, F.; Menziani, M. C.; Adamo, C. Optical properties of the dibenzothiazolylphenol molecular crystals through ONIOM calculations: the effect of the electrostatic embedding scheme. Theor. Chem. Acc. 2016, 135, 86.

(36) Wilbraham, L.; Coudert, F.-X.; Ciofini, I. Modelling photophysical properties of metal-organic frameworks: A Density Functional Theory based approach. Phys. Chem. Chem. Phys. 2016, 18, 25176-25182. 
(37) Meymand, F. M.; Mazhar, M.; Abdouss, M. Investigation of substituent effect on cool activity of perylene bisimide pigments. $J$. Coat. Technol. Res. 2019, 16, 439-447.

(38) Mazhar, M.; Abdouss, M.; Gharanjig, K.; Teimuri-Mofrad, R. Synthesis, characterization and near infra-red properties of perylenebisimide derivatives. Prog. Org. Coat. 2016, 101, 297-304.

(39) Zhang, F.; Ma, Y.; Chi, Y.; Yu, H.; Li, Y.; Jiang, T.; Wei, X.; Shi, J. Self-assembly, optical and electrical properties of perylene diimide dyes bearing unsymmetrical substituents at bay position. Sci. Rep. 2018, 8, No. 8208 .

(40) Liu, J.; Lu, Y.; Liu, J.; Yang, X.; Yu, X. Investigation of near infrared reflectance by tuning the shape of $\mathrm{SnO}_{2}$ nanoparticles. J. Alloys Compd. 2010, 496, 261-264.

(41) Hasche, T.; Canzler, T. W.; Scholz, R.; Leo, K. Coherent phonons in quasi-one-dimensional organic crystals. J. Lumin. 2001, 94-95, 673-676.

(42) Würthner, F.; Saha-Moeller, C. R.; Fimmel, B.; Ogi, S.; Leowanawat, P.; Schmidt, D. Perylene Bisimide Dye Assemblies as Archetype Functional Supramolecular Materials. Chem. Rev. 2016, 116, 962-1052.

(43) Görl, D.; Zhang, X.; Wuerthner, F. Molecular Aggregates of Perylene Bisimide Dyes in Water. Angew. Chem., Int. Ed. 2012, 51, 6328-6348.

(44) Chen, Z.; Lohr, A.; Saha-Moeller, C. R.; Wuerthner, F. Selfassembled $\pi$-stacks of functional dyes in solution: structural and thermodynamic features. Chem. Soc. Rev. 2009, 38, 564-584.

(45) Würthner, F. Perylene bisimide dyes as versatile building blocks for functional supramolecular architectures. Chem. Commun. 2004, $1564-1579$.

(46) Prampolini, G.; Bellina, F.; Biczysko, M.; Cappelli, C.; Carta, L.; Lessi, M.; Pucci, A.; Ruggeri, G.; Barone, V. Computational design, synthesis, and mechanochromic properties of new thiophene-based $\pi$ conjugated chromophores. Chem. - Eur. J. 2013, 19, 1996-2004.

(47) Ciardelli, F.; Ruggeri, G.; Pucci, A. Dye-containing polymers: Methods for preparation of mechanochromic materials. Chem. Soc. Rev. 2013, 42, 857-870.

(48) Pucci, A.; Ruggeri, G. Mechanochromic polymer blends. J. Mater. Chem. 2011, 21, 8282-8291.

(49) Pucci, A.; Bizzarri, R.; Ruggeri, G. Polymer composites with smart optical properties. Soft Matter 2011, 7, 3689-3700.

(50) Pucci, A.; Biver, T.; Ruggeri, G.; Meza, L. I.; Pang, Y. Greenblue luminescence dichroism of cyano-containing poly $[$ ( $\mathrm{m}$-phenylene ethynylene)-alt-(p-phenylene ethynylene)] aggregates dispersed in oriented polyethylene. Polymer 2005, 46, 11198-11205.

(51) Le Bahers, T.; Adamo, C.; Ciofini, I. A Qualitative Index of Spatial Extent in Charge-Transfer Excitations. J. Chem. Theory Comput. 2011, 7, 2498-2506.

(52) Muniz-Miranda, F.; Menziani, M. C.; Pedone, A. Assessment of Exchange-Correlation Functionals in Reproducing the Structure and Optical Gap of Organic-Protected Gold Nanoclusters. J. Phys. Chem. C 2014, 118, 7532-7544.

(53) Muniz-Miranda, F.; Menziani, M. C.; Pedone, A. DFT and TDDFT Assessment of the Structural and Optoelectronic Properties of an Organic $-\mathrm{Ag}_{14}$ Nanocluster. J. Phys. Chem. A 2015, 119, 50885098.

(54) Muniz-Miranda, F.; Presti, D.; Menziani, M. C.; Pedone, A. Electronic and optical properties of the $\mathrm{Au}_{22}[1,8$-bis(diphenylphosphino) octane $]_{6}$ nanoclusters disclosed by DFT and TD-DFT calculations. Theor. Chem. Acc. 2016, 135, 5.

(55) Muniz-Miranda, F.; Pedone, A.; Battistelli, G.; Montalti, M.; Bloino, J.; Barone, V. Benchmarking TD-DFT against Vibrationally Resolved Absorption Spectra at Room Temperature: 7-Aminocoumarins as Test Cases. J. Chem. Theory Comput. 2015, 11, 53715384.

(56) Muniz-Miranda, F.; Menziani, M. C.; Pedone, A. On the optoelectronic properties of phosphine and thiolate-protected undecagold nanoclusters. Phys. Chem. Chem. Phys. 2014, 16, 18749-18758. 